\title{
Analyzing the Performance of an Inset Fed Sierpinski's Carpet Rectangular Microstrip Fractal Antenna
}

\author{
Snehi Saraswati \\ Sam Higginbottom \\ Institute of Agriculture, \\ Technology and Sciences \\ Department of Electronics and \\ Communication Engineering \\ Naini, Allahabad - 221007, \\ India
}

\begin{abstract}
There are needs of wireless communication systems in modern society. The required systems should have wider bandwidth, low profile multiband and several applications. The researchers have initiated research in number of directions. New approaches and recent researches can be made for the study of fractal shaped antenna elements. Traditional antennas operate at single or dual frequency bands and different antenna is required for different applications. In this paper, the Sierpinski's carpet microstrip fractal antenna is considered. The behaviour of Sierpinski's antenna related to the design procedure, numerical simulation using methods of moments, importance of IE3D softwere to simulate this antenna are investigated.
\end{abstract}

\section{General Terms}

Antenna, wireless communication, dielectric substrate, fractal antenna, voltage standing wave ratio

\section{Keywords}

$3^{\text {rd }}$ iteration, simulated frequency, Sierpinski's antenna, frequencyband, return loss, FR4, Silicon, Duroid 6006.

\section{INTRODUCTION}

In 1983 Mandelbrot has proposed a fractal antenna which is new class of geometry. Due to specific geometrical properties, fractal shape antennas are known as antennas having different and useful features. A fractal antenna has a fractal, self similar design which maximizes the length and increase the perimeter of material. This material enables to receive and transmit the electromagnetic radiation in given capacity. Fractal antennas have the properties of self similarity and space filling which are utilized in the design of antennas having multiband behaviour and miniaturization. An object has the character of self similarity if it is build of sub units and sub units of multiple levels.

There is a need of an antenna which is smaller in size, light weight and consuming minimum fuel. This type of surface mounted antennas are helpful in cars, satellites, trains, aircrafts etc. Thus, space filling property of fractal antenna is necessary and helpful in the miniaturization of antennas.

Designs and applications of Sierpinski's antennas are studied by several researchers like Yao $\mathrm{Na}$ and ShiXio- wei [1], Mohamad Kamal A.Rahim, Mohmad Zoinol Abidin Abd Aziz and Noorsaliza Abdullah [2], DH. Werner, P. L. Wernerand, A.J. Ferraro [3] and S B Kumar, P K Singhal [4]. Recently Snehi Saraswati and Neelesh Agrawal [5] have studied the improvement of the performance of microstrip patch antenna.
The purpose of this researcher paper is to design a Sierpinski's Carpet rectangular antenna to obtain maximum return loss and more applicable in wireless communication system.

\section{SIERPINSKI'S FRACTAL ANTENNA}

The roles of properties of similarities and shape filling are remarkable in the shape of Fractal antennas. Many fractal geometries are useful in the development of the designing of antennas. The Sierpinski's carpet antenna is initiated with a suitable square. The square is divided into nine smaller square having cut out the centre square.
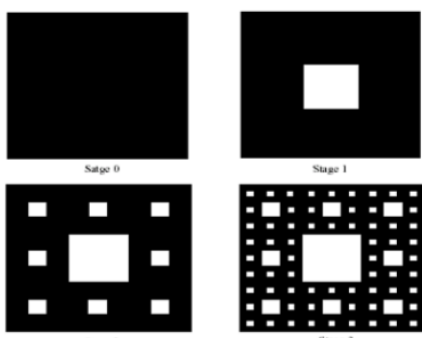

Figure 2.1

Figure 2.1 describes the design of Sierpinski's carpet antenna. The design of this antenna starts with a square in a plane and then it is divided into a small congruent squares where the open central square is dropped. The remaining each of eight squares is again divided into nine small congruent squares having each central square is dropped. This process is repeated several times as per our needs.

Fractal antenna has following advantages:

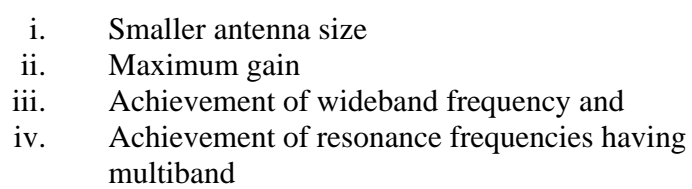

\subsection{Sierpinski's Carpet Antenna}

In 1916, Waelaw Sierpinski designed carpet antenna which is presently known as Sierpinski's carpet antenna. The designing of this antenna is followed by subdividing a shape into smaller copies of itself and removing one or more copies. This process can be continued as required to fulfill the need of our communication. One of several designs, one can be obtained by subdividing the rectangle into four rectangles and the removing the middle rectangle. Again dividing each of four rectangles into four rectangles, removing the middle rectangle 
in each case. The continuity of this process leads to the Sierpinski carpet.

The applications of fractal's have many benefits in development of various antenna elements. It has infinite complexity and self similarity while they are useful in designing antennas having very wideband performance.

\subsection{Design of Sierpinski's Carpet Antennas}

For the purpose of initial design, the square patch antenna is considered. The equations of microdtrip patch antenna are helpful in finding the dimensions of the antenna. The transmission line feeding technique is used to design of this antenna which is generally known as fractal design. Initially this antenna is designed upto second iteration. The following figure is helpful to understand the method pf designing Sierpinski's carpet antenna upto third iteration.

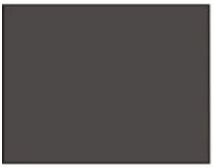

Zero iteration

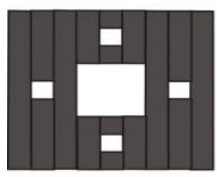

2nd iteration

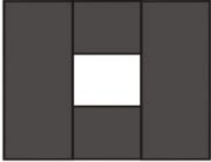

1st iteration

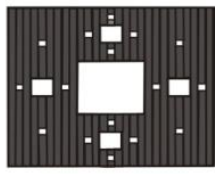

3rd iteration
Figure 2.2.1

Design and Fabrication of Microstrip Sierpinski Carpet Antenna and its applications

For our fractal de sign, this procedure has been generalized for rectangular Sierpinski's antenna as following.

Let us consider

$N_{n}$ be the number of black box,

$L_{n}$ be the ratio for the length,

$A_{n}$ be the ratio for the fractional areaafter the $\mathrm{n}^{\text {th }}$ iteration and $d_{n}$ is the capacity dimension.

$$
\begin{gathered}
N_{n}=4^{n}, L_{n}=\left(\frac{1}{3}\right)^{n}, A_{n}=\left(\frac{4}{5}\right)^{n} \\
d_{n}=\lim _{n \rightarrow \infty} \frac{n \log 4}{n \log \left(\frac{1}{3}\right)}=\frac{\log 4}{\log 3}=\frac{1.3862}{1.0986} \\
=1.2617
\end{gathered}
$$

\begin{tabular}{|l|l|l|l|l|}
\hline & $\begin{array}{l}\text { Zero } \\
\text { iteration }\end{array}$ & $\begin{array}{l}1^{\text {st }} \\
\text { iteration }\end{array}$ & $\begin{array}{l}2^{\text {nd }} \\
\text { iteration }\end{array}$ & $\begin{array}{l}3^{\text {rd }} \\
\text { iteration }\end{array}$ \\
\hline $\mathrm{N}_{\mathrm{n}}$ & 1 & 4 & 16 & 64 \\
\hline $\mathrm{L}_{\mathrm{n}}$ & 1 & 0.33 & 0.1111 & 0.0370 \\
\hline $\mathrm{A}_{\mathrm{n}}$ & 1 & 0.8 & 0.64 & 0.512 \\
\hline
\end{tabular}

For the successful designing of microstrip antenna,

For successful designing of microstrip antenna, there are needs of dielectric constant of $\operatorname{substrate}\left(\varepsilon_{\mathrm{r}}\right)$, the resonant frequency $\left(f_{o}\right)$ and height of substrate(h). Details of necessary informations may be devided by following formulae:

Width $\mathrm{W}$ is given by

$$
W=\frac{1}{2 f_{o} \sqrt{\mu_{o} \varepsilon_{o}}} \sqrt{\frac{2}{\varepsilon_{r}+1}}=\frac{v_{o}}{2 f_{o}} \sqrt{\frac{2}{\varepsilon_{r}+1}}
$$

Where $v_{o}$ is free- space velocity of light $=3 \times 10^{8} \mathrm{~m} / \mathrm{s}$.

The effective dielectric constantof microstrip patch antenna

$=\varepsilon_{e f f}=\frac{\varepsilon_{r}+1}{2}+\frac{\varepsilon_{r}-1}{2}\left[1+12 \frac{h}{W}\right]^{\frac{-1}{2}}$

Extension of length $=$

$$
\Delta L=0.412 h\left[\frac{\left(\varepsilon_{e f f}+0.3\right)\left(\frac{W}{h}+0.264\right)}{\left(\varepsilon_{e f f}-0.258\right)\left(\frac{W}{h}+0.8\right)}\right]
$$

\begin{tabular}{|c|c|c|c|c|c|c|}
\hline $\begin{array}{c}\text { Materia } \\
1\end{array}$ & $\begin{array}{l}\text { Design } \\
\text { ed } \\
\text { frequen } \\
\text { cy } \\
f_{0}(G H z)\end{array}$ & $\begin{array}{l}\text { Width(W)( } \\
\text { mm })\end{array}$ & $\varepsilon_{\text {reff }}$ & $\begin{array}{l}\Delta L \\
(\mathrm{~m} \\
\mathrm{m})\end{array}$ & $\begin{array}{l}L_{\text {eff }} \\
(\mathrm{m} \\
\mathrm{m})\end{array}$ & $\begin{array}{l}L \\
(\mathrm{~m} \\
\mathrm{m})\end{array}$ \\
\hline \multirow{5}{*}{$\begin{array}{c}\text { Substr } \\
\text { ate } \\
\text { FR: } 4 \\
\varepsilon_{\mathrm{r}}=4.4 \\
\mathrm{~h}= \\
1.61 \mathrm{~m} \\
\mathrm{~m}\end{array}$} & 2.5 & 36.51 & $\begin{array}{l}4.0 \\
7\end{array}$ & $\begin{array}{l}.74 \\
31\end{array}$ & $\begin{array}{l}29 . \\
74\end{array}$ & $\begin{array}{l}28 . \\
25\end{array}$ \\
\hline & 2.9 & 31.47 & $\begin{array}{l}4.0 \\
3\end{array}$ & $\begin{array}{l}.74 \\
13\end{array}$ & $\begin{array}{l}25 . \\
77\end{array}$ & $\begin{array}{l}24 . \\
29\end{array}$ \\
\hline & 3.3 & 27.66 & $\begin{array}{l}4.0 \\
0\end{array}$ & $\begin{array}{l}.73 \\
95\end{array}$ & $\begin{array}{l}22 . \\
73\end{array}$ & $\begin{array}{l}21 . \\
25\end{array}$ \\
\hline & 3.7 & 24.67 & $\begin{array}{l}3.9 \\
7\end{array}$ & $\begin{array}{l}.73 \\
77\end{array}$ & $\begin{array}{l}20 . \\
35\end{array}$ & $\begin{array}{l}18 . \\
87\end{array}$ \\
\hline & 4.1 & 22.27 & $\begin{array}{l}3.9 \\
4\end{array}$ & $\begin{array}{l}.73 \\
59\end{array}$ & $\begin{array}{l}18 . \\
43\end{array}$ & $\begin{array}{l}16 . \\
96\end{array}$ \\
\hline \multirow{5}{*}{$\begin{array}{c}\text { Substr } \\
\text { ate } \\
\text { DURO } \\
\text { ID } \\
6006 \\
\varepsilon_{\mathrm{r}}=6 \\
\mathrm{~h}= \\
1.61 \mathrm{~m} \\
\mathrm{~m}\end{array}$} & 2.5 & 32.07 & $\begin{array}{l}5.4 \\
7\end{array}$ & $\begin{array}{l}.71 \\
53\end{array}$ & $\begin{array}{l}25 . \\
65\end{array}$ & $\begin{array}{l}24 . \\
22\end{array}$ \\
\hline & 2.9 & 27.64 & $\begin{array}{l}5.4 \\
2\end{array}$ & $\begin{array}{l}.71 \\
31\end{array}$ & $\begin{array}{l}22 . \\
22\end{array}$ & $\begin{array}{l}20 . \\
79\end{array}$ \\
\hline & 3.3 & 24.30 & $\begin{array}{l}5.3 \\
7\end{array}$ & $\begin{array}{l}.71 \\
09\end{array}$ & $\begin{array}{l}19 . \\
62\end{array}$ & $\begin{array}{l}18 . \\
20\end{array}$ \\
\hline & 3.7 & 21.66 & $\begin{array}{l}5.3 \\
2\end{array}$ & $\begin{array}{l}.70 \\
87\end{array}$ & $\begin{array}{l}17 . \\
58\end{array}$ & $\begin{array}{l}16 . \\
16\end{array}$ \\
\hline & 4.1 & 19.56 & $\begin{array}{l}5.2 \\
7\end{array}$ & $\begin{array}{l}.70 \\
67\end{array}$ & $\begin{array}{l}15 . \\
94\end{array}$ & $\begin{array}{l}14 . \\
53\end{array}$ \\
\hline \multirow{5}{*}{$\begin{array}{c}\text { Substr } \\
\text { ate } \\
\text { SILIC } \\
\text { ON } \\
\varepsilon_{\mathrm{r}}=11.9 \\
\mathrm{~h}= \\
1.61 \mathrm{~m} \\
\mathrm{~m}\end{array}$} & 2.5 & 23.62 & $\begin{array}{l}10 . \\
49\end{array}$ & $\begin{array}{l}.67 \\
53\end{array}$ & $\begin{array}{l}18 . \\
53\end{array}$ & $\begin{array}{l}17 . \\
18\end{array}$ \\
\hline & 2.9 & 20.37 & $\begin{array}{l}10 . \\
35\end{array}$ & $\begin{array}{l}.67 \\
21\end{array}$ & $\begin{array}{l}16 . \\
08\end{array}$ & $\begin{array}{l}14 . \\
74\end{array}$ \\
\hline & 3.3 & 17.90 & $\begin{array}{l}10 . \\
23\end{array}$ & $\begin{array}{l}.66 \\
89\end{array}$ & $\begin{array}{l}14 . \\
21\end{array}$ & $\begin{array}{l}12 . \\
87\end{array}$ \\
\hline & 3.7 & 15.96 & $\begin{array}{l}10 . \\
12\end{array}$ & $\begin{array}{l}.66 \\
58 \\
\end{array}$ & $\begin{array}{l}12 . \\
74\end{array}$ & $\begin{array}{l}11 . \\
41\end{array}$ \\
\hline & 4.1 & 14.41 & $\begin{array}{l}10 . \\
01\end{array}$ & $\begin{array}{l}.66 \\
27 \\
\end{array}$ & $\begin{array}{l}11 . \\
56\end{array}$ & $\begin{array}{l}10 . \\
23\end{array}$ \\
\hline
\end{tabular}

(3) The actual length of the patch

$$
\begin{aligned}
L & =\frac{1}{2 f_{o} \sqrt{\varepsilon_{\text {eff }}} \sqrt{\mu_{o} \varepsilon_{o}}}-2 \Delta L \\
L_{\text {eff }} & =\frac{v_{0}}{2 f_{0} \sqrt{\varepsilon_{\text {reff }}}}
\end{aligned}
$$

(5) 


\subsection{Substrates}

In the paper Sierpinski's Carpet rectangular microstrip patch antenna has been designed using three different substrates and the results are compared.

\subsubsection{FR4}

This is a type of grade designation assigned to glass reinforced epoxy laminated sheets, rods, tubes and PCB(printed Circuit Boards) . The dielectric constant of it is equal to 4.4. Woven fiberglass cloth an epoxy resinbinder are composed into FR4. It has character of flame resistance/ self extinguishing. FR4 glass epoxy is popular having versatile enough pressure thermoset plastic laminate grade. It has a good strength to weight ratios and used as an electrical insulator with considerable mechanical strength.

\subsubsection{Silicon}

Mostly semiconductor industries product silicon wafers. It used as a substrate in microeletronic devices. The dielectric constant of this materials 11.9. This substrate is useful in case of an antenna with minimum dimensions.

\subsubsection{Duroid 6006:}

It is a composed microwave laminate and designed for microwave circuit and electronic applications which require a high dielectric constant. Duroid laminate is available with a dielectric constant value 6.00. This is easy in fabrication and stable in use. It has light dielectric constant, thickness control, good thermal mechanical stability and low moisture absorption.

\section{COMPARISON OF DESIGNED FREQUENCY AND SIMULATED FREQUENCY}

\begin{tabular}{|c|c|c|}
\hline & $\begin{array}{l}\text { Designed } \\
\text { frequency }(\mathrm{GHz})\end{array}$ & $\begin{array}{l}\text { Simulated } \\
\text { frequency }(\mathrm{GHz})\end{array}$ \\
\hline \multirow{5}{*}{$\begin{array}{c}\text { FR } 4 \\
\varepsilon_{\mathrm{r}}=4.4\end{array}$} & 2.5 & 2.47 \\
\hline & 2.9 & 2.86 \\
\hline & 3.3 & 3.25 \\
\hline & 3.7 & 3.68 \\
\hline & 4.1 & 4.07 \\
\hline \multirow{5}{*}{$\begin{array}{l}\text { DUROID - } \\
\quad 6006\end{array}$} & 2.5 & 2.41 \\
\hline & 2.9 & 2.88 \\
\hline & 3.3 & 3.27 \\
\hline & 3.7 & 3.62 \\
\hline & 4.1 & 4.02 \\
\hline & & \\
\hline
\end{tabular}

\section{VSWR (VOLTAGE STANDING WAVE RATIO ) AND RL(RETURN LOSS) :}

VSWR is the ratio of the maximum to minimum voltage of the Sierpinski's antenna.

The reflection coefficient $\rho$ is the ratio of incident wave amplitude $V_{i}$ and reflected voltage wave amplitude $V_{r}$ i.e. $\rho=\frac{V_{i}}{V_{r}}$.

If

$r=\frac{Z_{\text {input }}-Z_{0}}{Z_{\text {input }}+Z}$,

where $Z_{0}=$ characteristic impedance of the antenna.

Then

$\rho=|r|=\frac{V S W R-1}{V S W R+1}$

and

$$
V S W R=\frac{|r|+1}{|r|-1},|r| \neq 1 .
$$

The Characteristic $Z_{0}$ is calculated by

$$
Z_{0}=\sqrt{\frac{L}{C}}
$$

where $L=$ The inductance of the antenna

$\mathrm{C}=$ Capacitance of antenna which can be calculated by

$$
\begin{aligned}
& C=\frac{2 \pi \varepsilon}{\ln \left(\frac{a+h}{a}\right)}\left(\frac{W}{2 \pi \varepsilon}\right), \\
& L=\frac{\mu}{2 \pi} \ln \left(\frac{a+h}{a}\right)\left(\frac{W}{2 \pi \varepsilon}\right) .
\end{aligned}
$$

Hence, the characteristic impedance $Z_{0}$ is obtained by

$$
Z_{0}=\frac{1}{2 \pi} \sqrt{\frac{\mu}{\varepsilon}} \ln \left(\frac{a+h}{a}\right) .
$$

The return loss $S_{11}$ is calculated by following equation

$$
S_{11}=-20 \log \left(\frac{V_{r}}{V_{i}}\right)=-20 \log \left(\frac{V S W R-1}{V S W R+1}\right)
$$

Also

$$
\begin{aligned}
& S_{11}=10 \log \left(\frac{P_{i}}{P_{r}}\right) \text {, where } \mathrm{P}_{\mathrm{i}} \text { is incident power \& } \mathrm{P}_{\mathrm{r}} \text { is reflected } \\
& \text { power } \\
& =10 \log \left(\frac{V_{i}^{2}}{V_{r}^{2}}\right)^{\text {(since } \left.P_{i} \square V_{i}^{2}\right)} \\
& =10 \log \left(\frac{V_{i}}{V_{r}}\right)^{2}=20 \log \left(\frac{V_{i}}{V_{r}}\right)=20 \log P
\end{aligned}
$$




\begin{tabular}{|c|c|c|c|}
\hline & P & $\begin{array}{c}\text { Reflected } \\
\text { Power(\%) }\end{array}$ & $\begin{array}{c}\text { Return } \\
\text { Loss(dB) }\end{array}$ \\
\hline 1.1 & .048 & .230 & 26.375 \\
\hline 1.6 & .231 & 5.34 & 12.73 \\
\hline 2.1 & .355 & 12.60 & 8.99 \\
\hline 2.6 & .444 & 19.71 & 7.05 \\
\hline 3.1 & .512 & 26.21 & 5.81 \\
\hline 3.6 & .565 & 31.92 & 4.96 \\
\hline 4.1 & .608 & 36.97 & 4.32 \\
\hline 4.6 & .643 & 41.34 & 3.84 \\
\hline 5.1 & .672 & 45.16 & 3.45 \\
\hline 5.6 & .697 & 48.58 & 3.14 \\
\hline 6.6 & .737 & 54.32 & 2.65 \\
\hline & & & \\
\hline
\end{tabular}

Reflected Power is equal to square of Reflection coefficient.

\section{SIMULATION RESULTS \\ $0^{\text {th }}$ Iteration}

\begin{tabular}{|c|c|c|c|c|}
\hline Band & $\mathbf{f}_{\mathbf{c}}(\mathbf{G H z})$ & $\mathbf{S}_{\mathbf{1 1}}(\mathbf{d B})$ & $\mathbf{B W}(\mathbf{M H z})$ & $\begin{array}{c}\text { Antenna } \\
\text { Efficiency(\%) }\end{array}$ \\
\hline $\mathrm{B}_{1}$ & 5.21 & -35.3 & 173.45 & 78.42 \\
\hline $\mathrm{B}_{2}$ & 7.70 & -11.70 & 146.10 & 56.37 \\
\hline
\end{tabular}

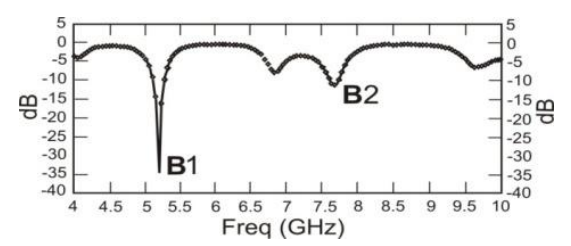

Figure 5.1

$\mathbf{1}^{\text {st }}$ Iteration

\begin{tabular}{|c|c|c|c|c|}
\hline Band & $\mathbf{f}_{\mathbf{c}}(\mathbf{G H z})$ & $\mathbf{S}_{\mathbf{1 1}}(\mathbf{d B})$ & $\mathbf{B W}(\mathbf{M H z})$ & $\begin{array}{c}\text { Antenna } \\
\text { Efficiency(\%) }\end{array}$ \\
\hline $\mathrm{B}_{1}$ & 4.69 & -26.04 & 102.38 & 78.37 \\
\hline $\mathrm{B}_{2}$ & 6.72 & -13.50 & 95.07 & 58.81 \\
\hline $\mathrm{B}_{3}$ & 7.90 & -13.28 & 162.08 & 58.35 \\
\hline
\end{tabular}

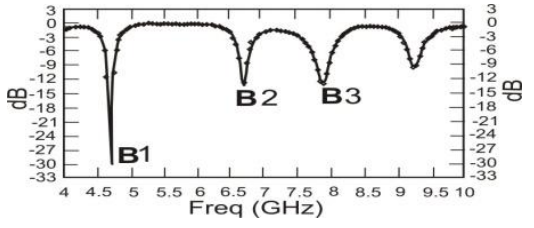

Figure 5.2

$2^{\text {nd }}$ Iteration

\begin{tabular}{|c|c|c|c|c|}
\hline Band & $\mathbf{f}_{\mathbf{c}}(\mathbf{G H z})$ & $\mathbf{S}_{\mathbf{1 1}}(\mathbf{d B})$ & $\mathbf{B W}(\mathbf{M H z})$ & $\begin{array}{c}\text { Antenna } \\
\text { Efficiency(\%) }\end{array}$ \\
\hline $\mathrm{B}_{1}$ & 4.64 & -20.87 & 84.51 & 78.23 \\
\hline $\mathrm{B}_{2}$ & 6.62 & -20.04 & 92.51 & 61.02 \\
\hline $\mathrm{B}_{3}$ & 7.86 & -11.12 & 119.31 & 56.69 \\
\hline $\mathrm{B}_{4}$ & 9.19 & -13.91 & 101.51 & 56.94 \\
\hline
\end{tabular}

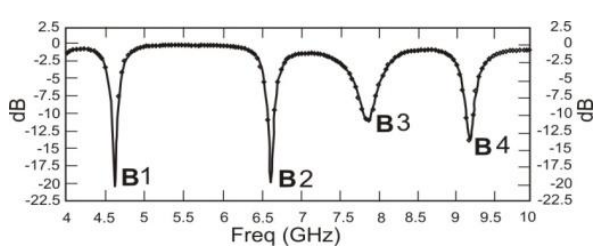

Figure 5.3

$3^{\text {rd }}$ iteration

\begin{tabular}{|c|c|c|c|c|}
\hline Band & $\mathbf{f}_{\mathbf{c}}(\mathbf{G H z})$ & $\mathbf{S}_{\mathbf{1 1}}(\mathbf{d B})$ & $\mathbf{B W}(\mathbf{M H z})$ & $\begin{array}{c}\text { Antenna } \\
\text { Efficiency(\%) }\end{array}$ \\
\hline $\mathrm{B}_{1}$ & 4.66 & -20.88 & 84.52 & 78.25 \\
\hline $\mathrm{B}_{2}$ & 6.64 & -20.05 & 92.53 & 61.04 \\
\hline $\mathrm{B}_{3}$ & 7.89 & -11.14 & 119.34 & 56.71 \\
\hline $\mathrm{B}_{4}$ & 9.20 & -13.93 & 101.55 & 56.95 \\
\hline
\end{tabular}

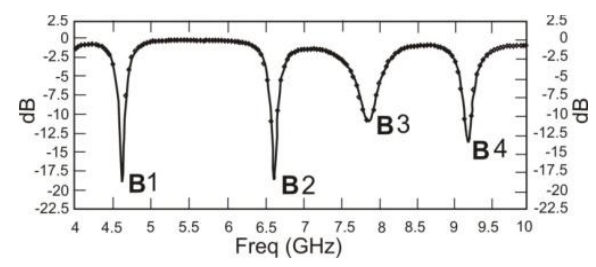

Figure 5.4

\section{DISCUSSIONS}

In the study of Sierpinski's carpet rectangular antenna following points are considered in detail:

1. The measurement of return loss follows the trend from simulation process.

2. This antenna gives a multiband frequency for the four iterations.

3. At higher frequency the percentage of bandwidth is increased.

4. Sierpinski's carpet antenna is considered as extension of conformal microstrip patch antenna. 


\section{CONCLUSIONS}

Following conclusions are derived :

1. An inset Sierpinski's carpet rectangular antenna is designed using fractal geometry/technique for multiband operation

2. The result of this paper indicate a desirable return loss and radiation pattern .

3. The geometry of this antenna describes multiband behaviour of the fractlal antenna and it describes multiband frequency but the frequency band is not predicted.

4. The possible bands for Sierpinski's rectangular antennas are narrowband.

5. By increasing the number of iterations, the number of frequency band s may be increasedas required.

6. Thus, an inset fed Sierpinski's carpet rectangular microstrip antenna is used for secure communication purpose.

7. Newly designedas well as proposed Sierpinski's antenna is best possible antenna.

\section{AKNOWLEDGEMENTS}

Author is grateful to her teachers Dr. Anil Kumar \&

Prof. A. K. Jaiswal, Head, Department of Electronic and Communication Engineering, Sam Higginbottom Institute of Agriculture, Technology and Sciences, Naini, Allahabad221007, India for suggesting the design and procedure to study an inset fed Sierpinski's carpet rectangular microstrip fractal antenna.

Author is grateful to the referee for his valuable suggestions and comments which improve the presentation of this research paper.

\section{REFERENCES}

[1] Yao Na and ShiXio- wei, "Analysis of multiband behaviour on Sierpinski's Carpet Fractal Antenna" National Laboratory of antennas and Microwave Technology, Xiadian University, APMC Proc 2005.

[2] Mohamad Kamal A.Rahim, Mohmad Zoinol Abidin Abd Aziz and Noorsaliza Abdullah, " Microstrip Sierpinski Carpet Antenna using Transmission Line Feeding , “ Wireless Communication Centre, APMC Proc 2005.

[3] DH. Werner, P. L. Wernerand, A.J. Ferraro, “ Frequency independent features of self similar fractal antenna, "In Proc IEEE AP- S Int. Symp., Baltimore, M D, July, 2126, 196, pp 2050- 2053.

[4] .S B Kumar , P K Singhal, “ ON THE INVESTIGATION OF A SIERPINSKI'S CARPET MICROSTRIP FRACTAL ANTENNA", INTERNATIONAL JOURNAL OF ENGINEERING SCIENCE \& TECHNOLOGY, Volume- 2, Issue- 2, 200203.

[5] Snehi Saraswati and Neelesh Agrawal, "Improvement of the Performance of Microstrip Patch Antenna", International Journal of Computer Applications(09758887), Volume 148- No. 10, August 2016. 